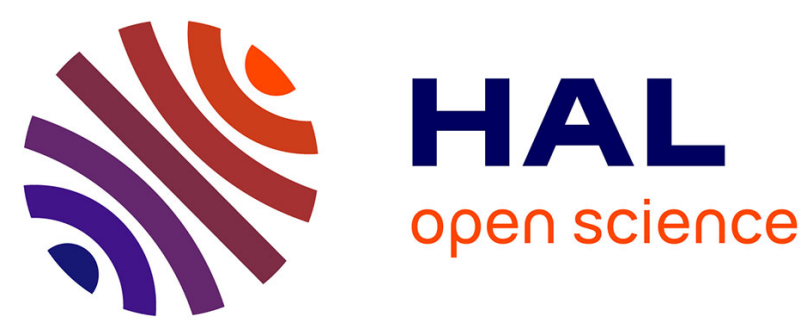

\title{
Effective bond orders from two-step spin-orbit coupling approaches: The I2, At2, IO+, and AtO+ case studies
}

Rémi Maurice, Florent Réal, Andre Severo Pereira Gomes, Valérie Vallet, Gilles F Montavon, Nicolas Galland

\section{- To cite this version:}

Rémi Maurice, Florent Réal, Andre Severo Pereira Gomes, Valérie Vallet, Gilles F Montavon, et al.. Effective bond orders from two-step spin-orbit coupling approaches: The I2, At2, IO+, and AtO+ case studies. Journal of Chemical Physics, 2015, 142, pp.094305. 10.1063/1.4913738 . in2p3-01123856

\section{HAL Id: in2p3-01123856 \\ https://hal.in2p3.fr/in2p3-01123856}

Submitted on 5 Mar 2015

HAL is a multi-disciplinary open access archive for the deposit and dissemination of scientific research documents, whether they are published or not. The documents may come from teaching and research institutions in France or abroad, or from public or private research centers.
L'archive ouverte pluridisciplinaire HAL, est destinée au dépôt et à la diffusion de documents scientifiques de niveau recherche, publiés ou non, émanant des établissements d'enseignement et de recherche français ou étrangers, des laboratoires publics ou privés. 


\title{
Effective bond orders from two-step spin-orbit coupling approaches: The $I_{2}, A t_{2}$, $\mathrm{IO}^{+}$, and $\mathrm{AtO}^{+}$case studies
}

\author{
Rémi Maurice, ${ }^{1}$ Florent Réal, ${ }^{2}$ André Severo Pereira Gomes, ${ }^{2}$ Valérie Vallet, ${ }^{2}$ Gilles \\ Montavon, ${ }^{1}$ and Nicolas Galland ${ }^{3}$ \\ 1) SUBATECH, CNRS UMR 6457, IN2P3/EMN Nantes/Université de Nantes, \\ 4 rue Alfred Kastler, BP 20722, 44307 Nantes Cedex 3, \\ France $^{\mathrm{a})}$ \\ 2) Laboratoire PhLAM, CNRS UMR 8523, Université de Lille, \\ 59655 Villeneuve d'Ascq Cedex, France \\ 3) CEISAM, UMR CNRS 6230, Université de Nantes, 2 rue de la Houssinière, \\ BP 92208, 44322 Nantes Cedex 3, France
}

(Dated: 16 February 2015)

The nature of chemical bonds in heavy main-group diatomics is discussed from the viewpoint of effective bond orders, which are computed from spin-orbit wave functions resulting from contracted spin-orbit configuration interaction calculations. The reliability of the relativistic correlated wave functions obtained in such two-step spinorbit coupling frameworks is assessed by benchmark studies of the spectroscopic constants with respect to either experimental data, or state-of-the-art fully relativistic correlated calculations. The $\mathrm{I}_{2}, \mathrm{At}_{2}, \mathrm{IO}^{+}$, and $\mathrm{AtO}^{+}$species are considered, and differences and similarities between the astatine and iodine elements are highlighted. In particular, we demonstrate that spin-orbit coupling weakens the covalent character of the bond in $\mathrm{At}_{2}$ even more than electron correlation, making the consideration of spin-orbit coupling compulsory for discussing chemical bonding in heavy $(6 p)$ main group element systems.

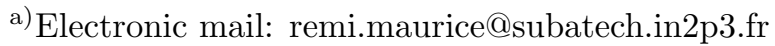




\section{INTRODUCTION}

Relativistic effects play a key role on molecular structures and properties, ${ }^{1}$ especially in the case of systems containing heavy atoms. Rationalizing the chemistry of such systems in terms of chemical bonding thus requires to introduce relativistic effects in the analysis. Our understanding of chemical bonding is usually based on atomic and molecular concepts. For instance, one can invoke molecular orbitals (MOs) expressed in terms of linear combinations of atomic orbitals (LCAOs), and assign for each MO a bonding, non-bonding or anti-bonding character, which leads to the concept of bond order (BO) or effective bond order (EBO). ${ }^{2}$ Population analyses defining atomic charges can also provide useful clues for rationalizing molecular structures and chemical bonds. Topological analyses of an appropriately chosen function are also particularly informative, namely that of the electron density in the quantum theory of atoms in molecules (QTAIM) fashion, ${ }^{3,4}$ or that of the electron localization function (ELF). ${ }^{5-9}$ All these tools help in understanding the chemistry with intuitive models, which is especially important for compounds involving elements whose chemical properties are poorly known.

Although astatine (At) is a member of the halogen family, many of the characteristics of this radioelement and its compounds remain elusive since all of its radionuclides are very short-lived. ${ }^{10}$ Understanding the chemical role of this element might not only be worthwhile from a fundamental viewpoint but also in view of the potential use of the ${ }^{211}$ At isotope in radiotherapy. ${ }^{11}$ Among the recent experimental highlights, it is worth quoting the determination of the ionization potential of the free atom, ${ }^{12}$ or the determination of predominance domains in the Pourbaix diagram $(\mathrm{E}-\mathrm{pH})$ of At in aqueous solution. ${ }^{13-15}$ One should also mention the theoretical prediction of a metallic behavior for condensed astatine, unlike the other halogens. ${ }^{16}$ Furthermore, stable At cationic forms $\left(\mathrm{At}^{+}\right.$and $\left.\mathrm{AtO}^{+}\right)$exist in aqueous solution and their coordination chemistry has been experimentally explored by reporting complexation constants with various inorganic ligands. ${ }^{17,18}$ As the longest-lived radionuclides ${ }^{210}$ At or ${ }^{211} \mathrm{At}$ can at present be produced only in trace quantities, it is not possible to obtain structural information from different spectroscopies. Therefore, the use of quantum chemical modeling methods offers the most promising way to shed light on astatine chemistry at a molecular scale.

Among the correlated relativistic electronic structure methods, contracted spin-orbit con- 
figuration interaction (c-SOCI) approaches are particularly interesting as they let us easily refer back to a spin-orbit free (SOF) picture of bonding while still providing accurate results, as attested by their success in computing zero-field splittings (ZFSs) and the electronic structure of $p, d$ and $f$ element containing systems. ${ }^{19-24}$ However, for the open-shell $6 p$ main group elements, treating spin-orbit coupling (SOC) a posteriori with a contracted scheme could be problematic ${ }^{25}$ whenever differential spin polarization of the $6 p_{1 / 2}$ and $6 p_{3 / 2}$ spinors has a strong impact on the electronic structure, as it is the case for $\mathrm{Tl}$ or $\mathrm{Bi}^{3+}$ for instance. ${ }^{26-29}$ In these cases, it is preferable to work with an uncontracted SOCI (uc-SOCI) correlated formalism rather than with the c-SOCI one, since we can retain the connection to the SOF picture while typically bringing the results closer to either experiment or fully relativistic correlated results.

The objective of this paper is to characterize, using two-step SOCI methods, the nature of the chemical bonds in heavy-element systems. Illustrations of the SOC effects in the $\mathrm{At}_{2}$ and $\mathrm{AtO}^{+}$molecules and in the lighter homologues $\mathrm{I}_{2}$, and $\mathrm{IO}^{+}$are given to support the originality of the proposed approach. Prior to discussing bonding, it is necessary to assess the accuracy of the two-step SOCI scheme in determining spectroscopic constants, such as bond lengths, harmonic frequencies, and dissociation energies, by comparing the results to those obtained with fully relativistic methods. After having validated our methodology, the chemical bonding will be analyzed in terms of EBOs. Note that EBOs have so far been defined in the framework of multiconfigurational non-relativistic or scalar-relativistic calculations. $^{2}$ This work thus represents a first attempt to extend the concept of EBO to relativistic calculations including SOC. By using two-step SOC approaches, the EBOs can be directly derived from the analysis of the resulting spin-orbit coupled wave functions, which leads, in a straightforward way, to a qualitative picture of the bonding. The stabilities of the interatomic bonds will also be quantified by computing the bond dissociation energies.

\section{THEORY AND METHODS}

\section{A. Effective bond orders}

Defining a $\mathrm{BO}$ from an $\mathrm{MO}$ point of view requires to assign a bonding, non-bonding or anti-bonding character to each MO. When only one electronic configuration is considered, the 
bond order is thus directly obtained from the half difference between the (total) occupation numbers of the bonding, $n^{\mathrm{b}}$, and anti-bonding, $n^{\mathrm{a}}$, orbitals:

$$
\mathrm{BO}=\frac{n^{\mathrm{b}}-n^{\mathrm{a}}}{2}
$$

In such a case, both $n^{\mathrm{b}}$ and $n^{\mathrm{a}}$ are integers, leading to integer or half-integer BOs. A more refined definition, allowing notably the EBO to progressively tend to zero when the bond is weakened up to dissociation, requires the use of multiconfigurational wave functions. ${ }^{2}$ One can thus define the EBO in terms of the natural orbital (NO) occupation numbers:

$$
\mathrm{EBO}=\frac{\sum_{b} \eta^{b}-\sum_{a} \eta^{a}}{2}
$$

where the indices $b$ and $a$ refer to bonding and anti-bonding active orbitals, respectively, and where $\eta^{b}$ and $\eta^{a}$ are the occupation numbers of the corresponding bonding and anti-bonding active orbitals. In practice, it is equivalent to compute EBOs by determining the $\mathrm{BO}$ of each of the configuration state function (CSF), and then summing the weighted BOs:

$$
\mathrm{EBO}=\sum_{i} \omega_{i} \frac{n_{i}^{\mathrm{b}}-n_{i}^{\mathrm{a}}}{2}
$$

where the index $i$ refers to CSFs, $\omega_{i}$ is the weight of the CSF $i$ in the wave function of interest, and where $n_{i}^{\mathrm{b}}$ and $n_{i}^{\mathrm{a}}$ are the number of bonding and anti-bonding electrons of the CSF $i$, respectively. In the general case, EBOs are neither integers nor half-integers. Note that the analysis only involves the active electrons, since it is assumed that the active space is properly chosen, such as bonding and anti-bonding orbitals are included pair-wise in the active space, and that the inactive electrons do not contribute directly to the bonding.

Defining $\sigma, \pi$ or $\delta$ type bonds also relies on a strict (or nearly ideal) separation of the $\sigma$, $\pi$ or $\delta$ type orbitals (by symmetry or by being close to a symmetrical situation). In a nonrelativistic or scalar relativistic context, all these conditions can be fulfilled in diatomics, and also in some binuclear compounds (containing two relatively heavy atoms). In more complex situations, the nature of the active orbitals should be carefully checked, and it is not guaranteed that EBOs can be properly defined from a standard LCAO calculation. One may however transform the active orbitals to "localize" them in terms of bonding, non-bonding and anti-bonding orbitals between the pair of atoms of interest. Such a discussion, although 
interesting, goes beyond the scope of the present work, aiming at introducing the concept of spin-orbit coupled EBO (SOC-EBO).

When two-step SOC calculations are performed, a set of SOF states is computed in the first step. A state-interaction (SI) matrix, built from the electronic energy matrix and the SOC matrix, can then be diagonalized within the basis of the spin components of the SOF states considered in the first step, i.e. a c-SOCI is often performed. Since the ground SOF and several excited SOF states are considered in the first step, state-averaged (SA) orbitals are usually built in order to ensure that the computed many-electron states are orthogonal. Of course, the determination of the spin-orbit free EBO (SOF-EBO) of a given SOF state cannot be obtained from the average occupation numbers of the SA orbitals, but it can in any case be done by using the CSF weights and BOs. Similarly, after the second step, one can obtain the SOC-EBO of a given spin-orbit (SO) state by computing the weighted sum of the SOF-EBOs of the spin components of each SOF state:

$$
\mathrm{SOC}-\mathrm{EBO}=\sum_{k} \omega_{k}\left[\sum_{i} \omega_{i, K} \frac{n_{i, K}^{\mathrm{b}}-n_{i, K}^{\mathrm{a}}}{2}\right]
$$

where $k$ refers to the spin components of a given SOF state $K, i$ refers to the CSFs used to express $K, \omega_{k}$ is the weight of the spin component of $K$ in the SO wave function of interest, where $\omega_{i, K}$ is the weight of the CSF $i$ in the SOF wave function of $K$. One should note that if an uc-SOCI is performed, the SO wave functions are directly expressed in terms of determinants. In such a case, the determination of the SOC-EBO of a given SO state can be obtained from Eq. 3 (the index $i$ would refer in such a case to determinants and not to CSFs).

\section{B. Computational details}

To evaluate the sensitivity of the EBO analyses and spectroscopic constants to the atomic basis sets we have used two different types of relativistic atomic basis sets. The first one refers to the segmented all-electron relativistically contracted basis sets of valence triplezeta quality with polarization functions adapted to the Douglas-Kroll Hess Hamiltonian ${ }^{30-32}$ (SARC-DKH-TZVP). ${ }^{33,34}$ For the oxygen and iodine atoms, the basis sets have been obtained by recontracting triple-zeta split-valence Karlsruhe non-relativistic basis sets ${ }^{35,36}$ under the influence of scalar relativistic effects. The following contraction schemes are used: 
(29s21p15d11f)/22s14p9d4f for At, (19s15p10d)/12s10p6d for I, and (11s6p1d)/6s3p1d for O. Note that the addition of $f$ polarization functions would slightly improve the computed spectroscopic constants of the iodine systems. Also, note that SARC-DKH basis sets are meant to be used for scalar relativistic calculations, or two-step SOC approaches, but not in two-component frameworks. The calculations with the SARC-DKH basis sets were performed with the ORCA program package. ${ }^{37}$ The second type of basis sets we have used corresponds to the all-electron atomic natural orbitals relativistic core correlation basis sets of quadruple zeta quality (ANO-RCC-QZP), ${ }^{38}$ with the following contraction schemes (25s22p16d12f4g)/9s8p6d4f3g, (22s19p13d5f3g)/8s7p5d4f2g, (14s9p4d3f2g)/5s4p3d2f1g, for At, I, and O, respectively. For these calculations we have used the MOLPRO (version 2012.1) ${ }^{39}$ and MOLCAS (version 7.8) ${ }^{40}$ quantum chemistry packages. As we shall discuss later both SARC-DKH-TZVP and ANO-RCC-QZP basis sets lead to similar EBOs, but the ANO-RCC-QZP basis sets appears to perform better for spectroscopic constants in the studied cases, in particular for bond dissociation energies and excitation energies.

In the first step of the calculation, SA complete active space self-consistent field (SACASSCF $)^{41,42}$ calculations are performed, in which the valence $p$ electrons are active. For the free atoms, free ions, and homonuclear diatomics, all the SOF states that can be built with the corresponding active spaces were considered (see Table I). The SA space is built by considering the $M_{S}(\max )$ components of each SOF state, and equal weights are considered for all SOF roots. In the considered heteronuclear diatomics, $\mathrm{IO}^{+}$and $\mathrm{AtO}^{+}$, this would lead to a too large number of SOF states, and potentially to averaging artifacts. Thus, we chose to restrict the set of states to the most important lowest-lying SOF states, that is including the singly-excited SOF states with respect to the ground state, and consistently truncating both SA and SI spaces based on an energetic criteria. In this way, we ensure that the $\pi$ symmetry is maintained, and the excited spin-components that couple most with the components of the ground SOF state are considered in the SI calculation. Note that the SI space is defined by the spin components of the SOF states considered in the first step. Contrary to a previous study on $\mathrm{IO}^{+}$and $\mathrm{AtO}^{+},{ }^{43}$ two quintet spin roots are considered in the averaging and their spin components included in the SOC calculation (see Table I). However, since the quintet states appear at excitation energies of about 4-5 eV, they do not influence much the ZFS values between the $\mathrm{X}^{+}$and the $a 1$ SO states; the ZFS values reported in Table VIII) are quite similar to the ones reported in Ref. 43. 
TABLE I. Active spaces, state-average (SA) and state-interaction (SI) spaces considered in the SOF and SOC calculations. For the SA and SI spaces, S refers to singlet, D to doublet, T to triplet, and Q to quintet spin states or their components, respectively.

\begin{tabular}{ccc}
\hline \hline $\mathrm{X}$ & Active Space (el./orb.) & SA/SI Spaces \\
\hline $\mathrm{O}$ & $4 / 3$ & $3 \mathrm{~T}, 6 \mathrm{~S}$ \\
$\mathrm{I} / \mathrm{At}$ & $5 / 3$ & $3 \mathrm{D}$ \\
$\mathrm{I}^{+} / \mathrm{At}^{+}$ & $4 / 3$ & $3 \mathrm{~T}, 6 \mathrm{~S}$ \\
$\mathrm{I}_{2} / \mathrm{At}_{2}$ & $10 / 6$ & $15 \mathrm{~T}, 21 \mathrm{~S}$ \\
$\mathrm{IO}^{+} / \mathrm{AtO}^{+}$ & $8 / 6$ & $2 \mathrm{Q}, 9 \mathrm{~T}, 6 \mathrm{~S}$ \\
\hline \hline
\end{tabular}

In the second step of the calculation, the SOC is introduced by diagonalizing the SI matrix corresponding to the electronic energy plus SOC matrix (c-SOCI scheme). A mean-field SOC operator is considered. ${ }^{44,45}$ As proposed by Teichteil et al. ${ }^{46}$ and Llusar et al. ${ }^{47}$ correlated electronic energies can be placed on the diagonal of the SI matrix. Here, $n$-electron valence states second-order perturbation theory (NEVPT2) ${ }^{48}$ correlated energies are used (within the strongly contracted scheme with the SARC-DKH basis sets and the partially contracted one for the ANO-RCC basis sets). The NEVPT2 method uses the Dyall's zeroth-order Hamiltonian, ${ }^{49}$ and (usually) does not suffer from intruder states. The core orbitals were kept frozen in the perturbative calculations with ANO-RCC basis sets, i.e. the $1 s$ orbitals of the $\mathrm{O}$ centers, all the orbitals lower in energy than the $5 \mathrm{~s}$ ones of the I centers (the $4 s$, $4 p$, and $4 d$ orbitals being frozen), and all the orbitals lower in energy than the 6-shells of the At centers (the $5 s, 5 p$, and $5 d$ ones being frozen).

For the considered diatomics the equilibrium distances and harmonic frequencies are computed using least-square fits of $a b$ initio values obtained every $0.01 \AA$ against an harmonic potential, following Hooke's law. The SOF and SOC electronic dissociation energies are computed considering the $\mathrm{X}_{2} \rightarrow \mathrm{X}+\mathrm{X}$, and $\mathrm{XO}^{+} \rightarrow \mathrm{X}^{+}+\mathrm{O}$ dissociation reactions, for which all the species are considered in their ground SOF or SOC state, respectively. The $\mathrm{C}_{1}$ symmetry point group is considered for the free atom and ion calculations to average the various spatially degenerate components of the atomic spin-orbit free states, while the $\mathrm{D}_{2 \mathrm{~h}}$ and $\mathrm{C}_{2 \mathrm{v}}$ point groups are considered for the $\mathrm{X}_{2}$ and $\mathrm{XO}^{+}$cases, respectively.

In some cases, due to the absence of available reference values in the literature, additional 
calculations have been performed to assess the accuracy of the results obtained with the aforementioned methods. In order to investigate the effect of $\mathrm{SO}$ polarization in the two-step SOC framework, we have used an uc-SOCI scheme, which diagonalizes the total relativistic Hamiltonian over a configurational space corresponding to the CAS plus all single-excitations (directly coupled by the effective one-electron mean-field spin-orbit operator ${ }^{50}$ ), projecting the effect of dynamic correlation, in the present case the (partially contracted) NEVPT2 scheme ${ }^{51}$ onto that SOCI model space by an effective spin-orbit Hamiltonian. ${ }^{28,47}$ The ucSOCI calculations were performed with the EPCISO code, ${ }^{28}$ interfaced to the MOLCAS quantum chemistry package (version 7.8$).{ }^{40}$ To have a complete set of four-component correlated reference calculations we have used a development version of the DIRAC electronic structure code $^{52}$ to perform Dirac-Coulomb coupled cluster (DC-CC) or DC intermediate Hamiltonian Fock space coupled cluster (DC-IHFSCC) calculations, using the same basis sets and calculation settings as in a previous publication of some of us. ${ }^{43}$

The SOF-EBOs and SOC-EBOs are determined at the minimum of each method (CASSCF, NEVPT2, c-SOCI/CASSCF, and c-SOCI/NEVPT2). Since we used internally contracted versions of NEVPT2, the reported CASSCF and NEVPT2 SOF-EBOs are equivalent in single-point calculations. When SOC is considered, there is an interplay between dynamic correlation and SOC. However, since only the diagonal of the SI matrix is affected by electron correlation (the off-diagonal SOC matrix elements are computed with the CASSCF wave functions in both cases), the EBOs computed at the c-SOCI/CASSCF and c-SOCI/NEVPT2 levels are expected to be very similar.

For the $\mathrm{X}_{2}$ cases, the $\sigma, \pi, \pi^{*}$ and $\sigma^{*}$ MOs are separated by symmetry $\left(a_{\mathrm{g}}, b_{2 \mathrm{u}}+b_{3 \mathrm{u}}\right.$, $b_{2 \mathrm{~g}}+b_{3 \mathrm{~g}}$, and $b_{1 \mathrm{u}}$, respectively). In the $\mathrm{XO}^{+}$cases, there is no symmetry distinction between bonding and anti-bonding orbitals, although the $\sigma$ and $\pi$ systems are strictly separated (the $\sigma, \pi, \pi^{*}$ and $\sigma^{*}$ MOs have $a_{1}, b_{1}+b_{2}, b_{1}+b_{2}$, and $a_{1}$ symmetries, respectively). As a consequence, bonding and anti-bonding orbitals could (partly) rotate. However, since we only consider a limited set of SOF states, the bonding and anti-bonding orbitals are clearly separated in terms of average occupation numbers. Visualization of the active orbitals confirmed that each active orbitals have a clear bonding or anti-bonding character, and therefore there is no ambiguity in the determination of EBOs in the studied cases. 
TABLE II. Excitation energies between spin-orbit levels of the O, I and At free atoms obtained at various levels of theory and with different relativistic atomic basis sets.

\begin{tabular}{|c|c|c|c|c|c|c|c|}
\hline \multirow[b]{2}{*}{$\mathrm{X}$} & \multirow[b]{2}{*}{$\Delta E(\mathrm{eV})$} & \multicolumn{3}{|c|}{ c-SOCI } & \multirow{2}{*}{$\frac{\mathrm{uc}-\mathrm{SOCI}}{\mathrm{NEVPT} 2^{\mathrm{b}}}$} & \multirow[b]{2}{*}{ DC-IHFSCCSD } & \multirow[b]{2}{*}{ Expt. } \\
\hline & & $\mathrm{CASSCF}^{\mathrm{a}}$ & NEVPT2 $^{\mathrm{a}}$ & NEVPT2 ${ }^{\mathrm{b}}$ & & & \\
\hline \multirow[t]{4}{*}{$\mathrm{O}$} & ${ }^{3} \mathrm{P}_{2} \rightarrow{ }^{3} \mathrm{P}_{1}$ & 0.018 & 0.018 & 0.019 & 0.019 & - & $0.020^{53}$ \\
\hline & ${ }^{3} \mathrm{P}_{2} \rightarrow{ }^{3} \mathrm{P}_{0}$ & 0.027 & 0.027 & 0.028 & 0.028 & - & $0.028^{53}$ \\
\hline & ${ }^{3} \mathrm{P}_{2} \rightarrow{ }^{3} \mathrm{D}_{2}$ & 2.182 & 2.092 & 1.955 & 1.955 & - & $1.967^{53}$ \\
\hline & ${ }^{3} \mathrm{P}_{2} \rightarrow{ }^{1} \mathrm{~S}_{0}$ & 5.442 & 5.045 & 5.040 & 5.040 & - & $4.190^{53}$ \\
\hline I & ${ }^{2} \mathrm{P}_{3 / 2} \rightarrow{ }^{2} \mathrm{P}_{1 / 2}$ & \multicolumn{2}{|c|}{$0.844^{\mathrm{c}}$} & $0.864^{\mathrm{c}}$ & 0.878 & 0.941 & $0.943^{54}$ \\
\hline At & ${ }^{2} \mathrm{P}_{3 / 2} \rightarrow{ }^{2} \mathrm{P}_{1 / 2}$ & \multicolumn{2}{|c|}{$2.461^{\mathrm{c}}$} & $2.524^{\mathrm{c}}$ & 2.875 & 2.888 & - \\
\hline
\end{tabular}

a The SARC-DKH-TZVP basis sets are used.

b The ANO-RCC-QZP basis sets are used.

${ }^{\text {c }}$ c-SOCI/CASSCF and c-SOCI/NEVPT2 results are here equivalent.

\section{RESULTS AND DISCUSSIONS}

\section{A. Lowest energy levels of the free atoms and cations}

When heavy atoms are considered, the calculation of dissociation energies of $\mathrm{I}_{2}, \mathrm{At}_{2}, \mathrm{IO}^{+}$, and $\mathrm{AtO}^{+}$requires a proper determination of the electronic structure of free atoms or ions, to ensure that the energetic stabilization of the dissociated fragments induced by SOC is well estimated. Tables II and III gather the computed energies levels for the free atoms O, I, At and the cations $\mathrm{I}^{+}$, and $\mathrm{At}^{+}$.

As reported in Table II, the SO splitting of the ${ }^{3} \mathrm{P}$ state of the oxygen atom is well reproduced, as well as the ${ }^{3} \mathrm{P}_{2} \rightarrow{ }^{1} \mathrm{D}_{2}$ excitation energy, both with CASSCF and NEVPT2 electronic energies. The ${ }^{3} \mathrm{P}_{2} \rightarrow{ }^{1} \mathrm{~S}_{0}$ excitation energy is overestimated by $0.85 \mathrm{eV}$, but since this excited state is very high in energy, it is not problematic for the discussion of chemical bonding and dissociation energies.

Iodine and astatine both have a ${ }^{2} \mathrm{P}$ SOF ground state. In c-SOCI calculations, as the effect of the dynamic correlation (projected on the diagonal of the SI matrix), is identical for all the six fine-structure components of the ${ }^{2} \mathrm{P}$ SOF state, the c-SOCI/CASSCF and c-SOCI/NEVPT2 results are strictly equivalent. The SO splitting of iodine appears to be 
TABLE III. Excitation energies between spin-orbit levels of the $\mathrm{I}^{+}$and $\mathrm{At}^{+}$free ions obtained at various levels of theory with different relativistic atomic basis sets.

\begin{tabular}{|c|c|c|c|c|c|c|c|}
\hline \multirow[b]{2}{*}{$\mathrm{X}^{+}$} & \multirow[b]{2}{*}{$\Delta E(\mathrm{eV})$} & \multicolumn{3}{|c|}{ c-SOCI } & \multirow{2}{*}{$\frac{\text { uc-SOCI }}{\text { NEVPT2 }^{\mathrm{b}}}$} & \multirow[b]{2}{*}{ DC-IHFSCCSD } & \multirow[b]{2}{*}{ Expt. } \\
\hline & & $\mathrm{CASSCF}^{\mathrm{a}}$ & $\mathrm{NEVPT}^{\mathrm{a}}$ & $\mathrm{NEVPT}^{\mathrm{b}}$ & & & \\
\hline \multirow[t]{4}{*}{$\mathrm{I}^{+}$} & ${ }^{3} \mathrm{P}_{2} \rightarrow{ }^{3} \mathrm{P}_{0}$ & 0.804 & 0.806 & 0.822 & 0.822 & 0.853 & $0.799^{57}$ \\
\hline & ${ }^{3} \mathrm{P}_{2} \rightarrow{ }^{3} \mathrm{P}_{1}$ & 0.757 & 0.765 & 0.793 & 0.792 & 0.870 & $0.879^{58}$ \\
\hline & ${ }^{3} \mathrm{P}_{2} \rightarrow{ }^{1} \mathrm{D}_{2}$ & 1.902 & 1.797 & 1.681 & 1.680 & 1.827 & $1.702^{57}$ \\
\hline & ${ }^{3} \mathrm{P}_{2} \rightarrow{ }^{1} \mathrm{~S}_{0}$ & 4.087 & 4.027 & 3.999 & 3.997 & 4.031 & $3.658^{57}$ \\
\hline \multirow[t]{4}{*}{$\mathrm{At}^{+}$} & ${ }^{3} \mathrm{P}_{2} \rightarrow{ }^{3} \mathrm{P}_{0}$ & 1.353 & 1.334 & 1.347 & 1.347 & 1.324 & - \\
\hline & ${ }^{3} \mathrm{P}_{2} \rightarrow{ }^{3} \mathrm{P}_{1}$ & 2.489 & 2.533 & 2.578 & 2.920 & 2.984 & - \\
\hline & ${ }^{3} \mathrm{P}_{2} \rightarrow{ }^{1} \mathrm{D}_{2}$ & 3.443 & 3.298 & 3.346 & 3.676 & 3.780 & - \\
\hline & ${ }^{3} \mathrm{P}_{2} \rightarrow{ }^{1} \mathrm{~S}_{0}$ & 6.800 & 6.745 & 6.850 & 7.524 & 7.675 & - \\
\hline
\end{tabular}

a The SARC-DKH-TZVP basis sets are used.

b The ANO-RCC-QZP basis sets are used.

underestimated by $0.08-0.10 \mathrm{eV}$ with all the considered two-step approaches. This is in line with the result of Roos et al. ${ }^{38}(0.863 \mathrm{eV})$, who used a similar approach, or with the value obtained by Fleig et al. ${ }^{55}$ using a four-component CI scheme $(0.854 \mathrm{eV})$. At the c-SOCI level, the use of the ANO-RCC of quadruple zeta quality only slightly increases the ${ }^{2} \mathrm{P}_{3 / 2} \rightarrow{ }^{2} \mathrm{P}_{1 / 2}$ splitting with respect to the SARC basis of triple zeta quality. The contribution of spinpolarization effects is small for iodine, $0.014 \mathrm{eV}$, but sizable for astatine, $0.351 \mathrm{eV}$. While the uc-SOCI/NEVPT2 underestimates the SO splitting of iodine with respect to experiment or to the fully relativistic DC-IHFSCC calculations, the agreement is much better for astatine. Because they lack spin-polarization effects, c-SOCI results underestimate by $10 \%$ the SO splitting of both iodine and astatine atoms. Note that the two-component Kramers restricted CASSCF calculations of Kim and Lee ${ }^{56}$ nicely corroborate with our fully relativistic values in both the iodine and astatine cases.

Regarding the $\mathrm{I}^{+}$and $\mathrm{At}^{+}$cations which have a $p^{4}$ valence configuration, the most interesting feature is related to the second-order SOC that introduces deviations to the Landé's rules that manifests itself by an inversion between ${ }^{3} \mathrm{P}_{1}$ and ${ }^{3} \mathrm{P}_{0}$ levels. This inversion has been experimentally determined decades ago for the $\mathrm{I}^{+}$ion, ${ }^{58}$ but also in the isoelectronic Te 
atom. ${ }^{59}$ Note that it is also the case for the Po atom, ${ }^{60}$ which is isoelectronic with the $\mathrm{At}^{+}$ ion. In the case of $\mathrm{I}^{+}$, DC-IHFSCC is the only method that captures the correct state ordering, while all two-step SOC calculations (contracted and uncontracted) retain the Landé's ordering, but placing the ${ }^{3} \mathrm{P}_{1}$ and ${ }^{3} \mathrm{P}_{0}$ levels less than $0.05 \mathrm{eV}$ apart from each other. It is thus not expected to be problematic for our semi-quantitative purposes.

As for At, the $\mathrm{At}^{+}$spectrum is not known experimentally. The comparison of the c-SOCI/CASSCF and c-SOCI/NEVPT2 results reveals a small interplay between electron correlation and SOC. The magnitude of the SO splitting is twice as large in astatine than in iodine. This larger SO splitting, which translates into a larger difference between the $\langle r\rangle$ values for the $p_{3 / 2}$ spinors over the $p_{1 / 2}$ spinors, implies that the contribution of spin-polarization effects is way stronger in $\mathrm{At}^{+}$than in $\mathrm{I}^{+}$; this is the reason why the uc-SOCI/NEVPT2 results exhibit smaller deviations with respect to the DC-IHFSCC results than the c-SOCI/NEVPT2 ones. As in the case of At, the c-SOCI method underestimates by about 10\% the SO splittings, making (two-step) c-SOCI methods useful for semi-quantitative estimates of SOC consequences.

\section{B. $\mathrm{I}_{2}$ and $\mathrm{At}_{2}$}

\section{Ground-state bond lengths, harmonic frequencies, and dissociation energies}

The $\mathrm{I}_{2}$ and $\mathrm{At}_{2}$ systems have already been studied in the literature at various levels of theory. ${ }^{4,9,18,61-66}$ The DC-CCSD(T) calculations of Visscher and Dyall ${ }^{62}$ and recent twocomponent $\operatorname{CCSD}(\mathrm{T})$ calculations of Höfener et $a l .{ }^{66}$ are here taken as reference computational values.

In Table IV, we present the spectroscopic constants and bond dissociation energies of $\mathrm{I}_{2}$ and $\mathrm{At}_{2}$ computed without and with SOC and the ANO-RCC basis sets. Comparing first the results obtained at the SOF CASSCF and NEVPT2 levels, and at the corresponding c-SOCI ones, we note that the role of both dynamical electron correlation and SOC is larger in $\mathrm{At}_{2}$ than in $\mathrm{I}_{2}$. In both cases dynamic correlation strengthens the bond, while SOC weakens it:

for instance, in $\mathrm{At}_{2}$, the bond lengths decreases from $2.913 \AA$ to $2.849 \AA$ under the effect of dynamic correlation, and increases from $2.913 \AA$ to $3.108 \AA$ under the effect of SOC. Note 
TABLE IV. Ground-state bond lengths, harmonic frequencies, and dissociation energies of $\mathrm{I}_{2}$ and $\mathrm{At}_{2}$ obtained at various levels of theory with the ANO-RCC-QZP basis sets.

\begin{tabular}{ccccccc}
\hline \hline Method & \multicolumn{3}{c}{$\mathrm{I}_{2}$} & \multicolumn{3}{c}{$\mathrm{At}_{2}$} \\
& $r_{\mathrm{e}}(\AA)$ & $\omega_{\mathrm{e}}\left(\mathrm{cm}^{-1}\right)$ & $D_{\mathrm{e}}(\mathrm{eV})$ & $r_{\mathrm{e}}(\AA)$ & $\omega_{\mathrm{e}}\left(\mathrm{cm}^{-1}\right)$ & $D_{\mathrm{e}}(\mathrm{eV})$ \\
\hline CASSCF & 2.792 & 196 & 1.25 & 2.913 & 140 & 1.10 \\
NEVPT2 & 2.667 & 224 & 2.07 & 2.849 & 160 & 1.86 \\
c-SOCI/CASSCF & 2.748 & 185 & 0.77 & 3.108 & 81 & 0.17 \\
c-SOCI/NEVPT2 & 2.679 & 216 & 1.57 & 2.957 & 124 & 0.85 \\
uc-SOCI/NEVPT2 & 2.679 & 216 & 1.56 & 2.971 & 118 & 0.64 \\
DC-CCSD $(\mathrm{T})^{62}$ & 2.717 & 206 & 1.28 & 3.046 & 108 & 0.63 \\
X2C-CCSD $(\mathrm{T})^{66}$ a & 2.692 & 206 & 1.54 & 3.006 & 110 & 0.79 \\
Expt. ${ }^{67}$ & 2.666 & 214 & 1.56 & - & - & - \\
\hline \hline
\end{tabular}

${ }^{a}$ Complete basis set extrapolations were performed.

that these effects are not additive; the interplay between the dynamic correlation and the SOC leads to a clear stabilization of the I-I bond, while it destabilizes the At-At bond. This means that the role of SOC on the $\mathrm{At}_{2}$ bond is at least as important as that of dynamic correlation. This highlights the growing influence of SOC on the strength of chemical bonds as the atomic number increases.

As seen from Table IV and Table SI, ${ }^{68}$ the SARC-DKH-TZVP basis set yields spectroscopic constants that tend to underestimate the bond strengths in terms of distance, harmonic frequency and dissociation energy, as was also found by other authors with various methods. ${ }^{9,61-65}$ However, the use of ANO-RCC basis set on iodine centers yields data in excellent agreement not only with the experimental data ${ }^{67}$ but also the latest reference calculations reported by Höfener et al. ${ }^{66}$ with the two-component X2C-CCSD(T) method, and extrapolating the correlation energies to the complete basis set (CBS) limit. Therefore, we conclude that the ANO-RCC-QZP basis set tends to perform better than the SARC-DKH-TZVP one for our purposes, although such a good agreement obtained with respect to the reference values with the ANO-RCC-QZP basis set was not expected at the c-SOCI/NEVPT2 level. 
In order to assess the role of the interplay between electron correlation and SOC on the $\mathrm{I}_{2}$ and $\mathrm{At}_{2}$ spectroscopic constants, these quantities are also computed with an ucSOCI calculation (see Table IV). The effect of SO polarization is expected to be negligible for $\mathrm{I}_{2}$, contrary to the $\mathrm{At}_{2}$ case. The computed uc-SOCI spectroscopic data are in excellent agreement with the DC-CCSD $(\mathrm{T})$ and $\mathrm{X} 2 \mathrm{C}-\mathrm{CCSD}(\mathrm{T})$ results, noting however that the uc-SOCI/NEVPT2 bond dissociation energy is $0.16 \mathrm{eV}$ lower than the X2C-CCSD(T)/CBS value in $\mathrm{At}_{2}$. However, with respect to the discussion of the At-At chemical bond, the fact that the uc-SOCI/NEVPT2 computed bond lengths $\left(\Delta r_{e}=0.014 \AA\right)$ are not very different from the c-SOCI ones, suggests that the interplay between SOC and electron correlation is not crucial for the description of the chemical bonding in this molecule. Also, the effect of SO polarization on $D_{\mathrm{e}}$ is about $-0.21 \mathrm{eV}$, which almost matches twice the effect on the free atom $(-0.23 \mathrm{eV})$. Therefore, we conclude that the $\mathrm{SO}$ polarization is almost quenched in the molecule. This leads us to expect that the c-SOCI/NEVPT2 approach is sufficient for EBO analyses. The discrepancy between the two-step SOCI approaches and the two-component correlated value in $\mathrm{At}_{2}$ is thus not expected to be essentially related to SOC, but most probably origins from the limitations in the treatment of dynamic correlation associated with the second-order perturbation theory treatment.

\section{Effective bond orders}

Having validated our methodology, we now focus our analysis on EBOs for the $\mathrm{I}_{2}$ and $\mathrm{At}_{2}$ systems. The effective bond orders have been determined at the minimum of each method (see Table V). Since two-step approaches are usually used for single-point calculations at given references geometries, it may be informative to compare the values obtained at different internuclear distances. For $\mathrm{I}_{2}$, all the considered geometries differ by less than $0.1 \AA$, which does not lead to noticeable changes in the SOF and SO ground-state wavefunctions. Therefore, using equilibrium or fixed geometries does not significantly affect the

EBO value. It is interesting to note that, while the SARC-DKH-TZVP basis yielded a slightly smaller bond strength for $\mathrm{I}_{2}$ with respect to the ANO-RCC-QZP one, the resulting EBOs are very much the same (see Table V and Table SII ${ }^{68}$ ). One can note that the nondynamic correlation within the active space decreases the EBO from the single-determinantal picture (the lowest-energy electronic configuration is $\sigma^{2} \pi^{4} \pi^{* 4}$, leading to a BO of one) by 
TABLE V. Ground-state EBOs of $\mathrm{I}_{2}$ and $\mathrm{At}_{2}$ computed at various levels of theory at the corresponding equilibrium geometries with the ANO-RCC-QZP basis sets, unless specified otherwise.

\begin{tabular}{|c|c|c|c|c|c|c|c|c|}
\hline \multirow{2}{*}{ Method } & \multicolumn{4}{|c|}{$\mathrm{I}_{2}$} & \multicolumn{4}{|c|}{$\mathrm{At}_{2}$} \\
\hline & $\mathrm{r}_{\mathrm{e}}(\AA)$ & $\mathrm{EBO}_{\sigma}$ & $\mathrm{EBO}_{\pi}$ & $\mathrm{EBO}$ & $\mathrm{r}_{\mathrm{e}}(\AA)$ & $\mathrm{EBO}_{\sigma}$ & $\mathrm{EBO}_{\pi}$ & $\mathrm{EBO}$ \\
\hline CASSCF & 2.729 & 0.93 & 0.00 & 0.92 & 2.913 & 0.92 & 0.00 & 0.92 \\
\hline NEVPT2 & 2.667 & 0.93 & 0.00 & 0.93 & 2.849 & 0.93 & 0.00 & 0.93 \\
\hline c-SOCI/CASSCF & 2.748 & 0.91 & -0.01 & 0.90 & 3.108 & 0.75 & -0.07 & 0.68 \\
\hline c-SOCI/NEVPT2 $2^{\mathrm{a}}$ & 2.732 & 0.91 & 0.00 & 0.91 & 2.916 & 0.83 & -0.05 & 0.78 \\
\hline c-SOCI/NEVPT2 & 2.679 & 0.93 & 0.00 & 0.93 & 2.957 & 0.82 & -0.04 & 0.78 \\
\hline uc-SOCI/NEVPT2 & 2.679 & 0.89 & 0.00 & 0.89 & 2.971 & 0.75 & -0.08 & 0.66 \\
\hline
\end{tabular}

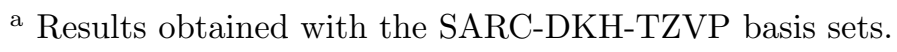

one order of magnitude more than the SOC. Thus, SOC has not much effect on EBOs in this case. On the contrary, SOC has a (twice) stronger effect than non-dynamical correlation in $\mathrm{At}_{2}$. The c-SOCI EBOs in $\mathrm{At}_{2}, 0.78$, is significantly smaller than in $\mathrm{I}_{2}(0.93)$.

In Table $\mathrm{V}$, we have divided the EBO values into contributions arising from the $\sigma$ and $\pi$ systems. We can scrutinize the influence of SOC on the EBO of the homonuclear diatomics by noting that SOC makes the ground state of these molecules (especially for $\mathrm{At}_{2}$ ) less $\sigma$ bonding and slightly more $\pi$ anti-bonding. This behavior can be rationalized by analyzing the dominant configurations of the relevant SOF and SO wave functions. The groundstate SOF wave function is dominated by the $\sigma^{2} \pi^{4} \pi^{* 4}$ configuration (which, as mentioned previously, corresponds to the ground state in a single-determinantal picture). Since this SOF ground state is a spin-singlet state and only singlet and triplet spin states can be built with the considered active space, the SO stabilization of the ground state is here essentially related to the coupling with spin components of singly-excited triplet configurations with respect to the dominant SOF configuration of the ground state. Moreover, the SOC cannot break the inversion symmetry, i.e. only $g \rightarrow g$ and $u \rightarrow u$ excitations are symmetry allowed. Only four configurations fulfill these requirements, all of them belonging to the $\sigma^{2} \pi^{3} \pi^{* 4} \sigma^{* 1}$ electronic configuration, and have an $\left|M_{S}\right|$ value of 1 . These (non-bonding) configurations 
are less $\sigma$ bonding than the ground SOF configuration (their $\sigma$ BO being 0.5), while being $\pi$ anti-bonding (their $\pi \mathrm{BO}$ being -0.5). Therefore, the ground SO state becomes significantly less bonding in terms of EBO than the ground SOF state. In other words, SOC weakens the covalent character of the bond in $\mathrm{At}_{2}$.

This result is in agreement with previous analyses based on the topological analyses performed on top of two-component density functional theory (DFT) calculations, ${ }^{4,9}$ and also discussions based on the concept of orbital hybridizations or of coupled molecular spinors. ${ }^{62,65}$ Note that the contraction partly inhibit the mixing of the $\sigma^{2} \pi^{4} \pi^{* 4}$ and $\sigma^{2} \pi^{3} \pi^{* 4} \sigma^{* 1}$ configurations, and therefore uc-SOCI calculations leads to slightly smaller EBOs in $\mathrm{I}_{2}(0.89)$ and $\mathrm{At}_{2}(0.66)$ than the c-SOCI ones. However, the qualitative difference between the iodine and astatine compounds can already be quantified at the c-SOCI level. Therefore, we conclude that the comparison of SOF-EBOs and SOC-EBOs is also another way of highlighting the important role of $\mathrm{SOC}$ on the chemical bond in $\mathrm{At}_{2}$.

\section{C. $\mathrm{IO}^{+}$and $\mathrm{AtO}^{+}$}

\section{Ground-state bond lengths, harmonic frequencies, and dissociation energies}

Depending on the $\mathrm{pH}$ and the potential $\mathrm{E}$, the $\mathrm{AtO}^{+}$species can be thermodynamically stable in aqueous solution. ${ }^{13}$ The ground state in the gas phase is expected to have a dominant spin-triplet character, while the ground state in solution was predicted to be dominated by a spin-singlet character based on two-component DFT calculations. ${ }^{69,70}$ Very recently, gas-phase reference calculations were also reported ${ }^{43}$ providing us with reference values to compare with. In Ref. 43, multireference calculations DC-IHFSCC calculations were performed on $\mathrm{IO}^{+}$and $\mathrm{AtO}^{+}$, confirming that both molecules have a (relativistic) ground state dominated by one single configuration. For consistency, we only report in Table VI the DC-CCSD $(\mathrm{T})$ values, which are very similar to the DC-IHFSCC ones. Note that, since $\mathrm{DC}-\mathrm{CCSD}(\mathrm{T})$ energies for the free $\mathrm{O}$ atom cannot be properly computed with the current implementation of DIRAC, no reference $D_{e}$ values exist for $\mathrm{IO}^{+}$and $\mathrm{AtO}^{+}$.

As can be seen in Table VI, the $r_{\mathrm{e}}$ and $\omega_{\mathrm{e}}$ values computed with the c-SOCI/NEVPT2 method agree well with the $\mathrm{DC}-\mathrm{CCSD}(\mathrm{T})$ results. It is interesting to note that in both the 
TABLE VI. Ground-state bond lengths, harmonic frequencies, and dissociation energies of $\mathrm{IO}^{+}$ and $\mathrm{AtO}^{+}$computed at various levels of theory with the ANO-RCC-QZP basis sets.

\begin{tabular}{cccccccc}
\hline \hline \multirow{2}{*}{ Method } & \multicolumn{3}{c}{$\mathrm{IO}^{+}$} & & \multicolumn{3}{c}{$\mathrm{AtO}^{+}$} \\
\cline { 2 - 4 } CASSCF & 1.869 & 708 & 2.10 & & 1.970 & 638 & 1.74 \\
NEVPT2 & 1.797 & 877 & 3.60 & & 1.892 & 792 & 3.15 \\
c-SOCI/CASSCF & 1.872 & 695 & 1.79 & & 1.979 & 605 & 1.08 \\
c-SOCI/NEVPT2 & 1.799 & 870 & 3.26 & & 1.900 & 786 & 2.43 \\
uc-SOCI/NEVPT2 & 1.798 & 874 & 3.25 & & 1.893 & 799 & 2.30 \\
DC-CCSD $(\mathrm{T}){ }^{43}$ & 1.829 & 763 & - & & 1.930 & 676 & - \\
\hline \hline
\end{tabular}

$\mathrm{IO}^{+}$and $\mathrm{AtO}^{+}$cases, dynamical correlation significantly strengthens the bond. Although SOC induces minor changes to the bond lengths and harmonic frequencies, it significantly reduces the dissociation energies for $\mathrm{IO}^{+}$and $\mathrm{AtO}^{+}$by $0.34 \mathrm{eV}$ and $0.72 \mathrm{eV}$, respectively. These contributions are of the same magnitude as those estimated for $I_{2}$ and $A t_{2}$. The SOC contribution to the dissociation energy arises from the difference of the SO energetic stabilizations of (i) the diatomics of interest and (ii) the corresponding free heavy atoms and ions. In the iodine molecules, the SOC is essentially quenched at the equilibrium geometry, thus the decrease of the dissociation energies arise only from the atomic fragments. In astatine molecules, SOC coupling also contributes to the total ground-state equilibrium energy: if one neglects that contribution, $\mathrm{At}_{2}$ would for instance be only weakly bound, by no more than $0.2 \mathrm{eV}$, while it is actually bound by about $0.85 \mathrm{eV}$ at the c-SOCI/NEVPT2 level. In $\mathrm{AtO}^{+}$, the SOC stabilization of the ground state at the c-SOCI/NEVPT2 equilibrium geometry accounts for about half of $D_{e}$. As in the $\mathrm{I}_{2}$ and $\mathrm{At}_{2}$ homonuclear molecules, changing the atomic basis set to the smaller SARC-DKH-TZVP one has a minor effect on the bond distances and frequencies, but significantly decreases the $\mathrm{XO}^{+}$dissociation energies, by $0.93 \mathrm{eV}$ and $0.39 \mathrm{eV}$, respectively, at the c-SOCI/NEVPT2 level (see Table SIII $\left.^{68}\right)$. The good accuracy of the results obtained with the ANO-RCC-QZP basis sets for the homonuclear molecules makes us think that the ANO-RCC-QZP basis sets may also yield (very) accurate dissociation energies for the $\mathrm{IO}^{+}$and $\mathrm{AtO}^{+}$systems. However, in the absence of experimental or reference data, one cannot definitely conclude on the quality of 
TABLE VII. Ground-state EBOs of $\mathrm{IO}^{+}$and $\mathrm{AtO}^{+}$obtained at various levels of theory at the corresponding equilibrium geometries with the ANO-RCC-QZP basis sets, unless specified otherwise.

\begin{tabular}{|c|c|c|c|c|c|c|c|c|}
\hline \multirow{2}{*}{ Method } & \multicolumn{4}{|c|}{$\mathrm{IO}^{+}$} & \multicolumn{4}{|c|}{$\mathrm{AtO}^{+}$} \\
\hline & $\mathrm{r}_{\mathrm{e}}(\AA)$ & $\mathrm{EBO}_{\sigma}$ & $\mathrm{EBO}_{\pi}$ & $\mathrm{EBO}$ & $\mathrm{r}_{\mathrm{e}}(\AA)$ & $\mathrm{EBO}_{\sigma}$ & $\mathrm{EBO}_{\pi}$ & $\mathrm{EBO}$ \\
\hline CASSCF & 1.869 & 0.92 & 0.84 & 1.76 & 1.970 & 0.90 & 0.82 & 1.72 \\
\hline NEVPT2 & 1.797 & 0.92 & 0.86 & 1.80 & 1.892 & 0.92 & 0.85 & 1.77 \\
\hline c-SOCI/CASSCF & 1.872 & 0.91 & 0.83 & 1.74 & 1.979 & 0.86 & 0.78 & 1.64 \\
\hline c-SOCI/NEVPT2 $2^{\mathrm{a}}$ & 1.844 & 0.91 & 0.84 & 1.75 & 1.921 & 0.89 & 0.82 & 1.70 \\
\hline c-SOCI/NEVPT2 & 1.799 & 0.93 & 0.85 & 1.78 & 1.900 & 0.87 & 0.79 & 1.66 \\
\hline uc-SOCI/NEVPT2 & 1.789 & 0.90 & 0.89 & 1.78 & 1.893 & 0.86 & 0.87 & 1.73 \\
\hline
\end{tabular}

${ }^{a}$ Results obtained with the SARC-DKH-TZVP basis sets.

the obtained results.

\section{Effective bond orders}

EBOs are presented for the ground SOF state $\left({ }^{3} \Sigma^{-}\right)$and the ground SO state $\left(\mathrm{X} 0^{+}\right)$ at the minimum of each method in Table VII (see Table SIV $^{68}$ for the results obtained with the SARC-DKH-TZVP basis sets). Note that Table VIII also provides EBOs for the lowest-energy excited SOF and SO states at the uc-SOCI geometries. By comparing these two tables, we conclude that the ground-state EBOs are not much affected by geometrical effects. Therefore, for the sake of simplicity, our analysis is essentially focussed on the results presented in Table VIII.

In order to understand the EBOs of the lowest-lying SO states, it is important to discuss their dominant components in terms of the lowest-lying SOF states. The $\mathrm{X}^{+} \mathrm{SO}$ state is essentially composed of the $\left|M_{S}\right|=0$ component of ${ }^{3} \Sigma^{-}$and of ${ }^{1} \Sigma^{+}$SOF states (about $92 \%$ and $7 \%$, respectively, for $\mathrm{IO}^{+}$, and about $69 \%$ and $26 \%$, respectively, for $\mathrm{AtO}^{+}$). Since ${ }^{3} \Sigma^{-}$ and ${ }^{1} \Sigma^{+}$have similar SOF-EBOs, SOC does not drastically change the EBO of the ground state. The doubly degenerate $a 1 \mathrm{SO}$ state is dominated by the $\left|M_{S}\right|=1$ components of ${ }^{3} \Sigma^{-}$, and also mix with some excited triplet configurations that exhibit a $\pi$ non-bonding 
TABLE VIII. Lowest-energy states vertical transition energies $\left(T_{v}\right)$ and $\mathrm{EBOs}$ of $\mathrm{IO}^{+}$and $\mathrm{AtO}^{+}$ obtained at various levels of theory with the ANO-RCC-QZP basis sets at the uc-SOCI/NEVPT2 equilibrium geometries $\left(r\left(\mathrm{IO}^{+}\right)=1.789 \AA\right.$ and $\left.r\left(\mathrm{AtO}^{+}\right)=1.893 \AA\right)$.

\begin{tabular}{|c|c|c|c|c|c|c|c|c|c|}
\hline \multirow{2}{*}{ Method } & \multirow{2}{*}{ State } & \multicolumn{4}{|c|}{$\mathrm{IO}^{+}$} & \multicolumn{4}{|c|}{$\mathrm{AtO}^{+}$} \\
\hline & & $T_{v}(\mathrm{eV})$ & $\mathrm{EBO}_{\sigma}$ & $\mathrm{EBO}_{\pi}$ & $\mathrm{EBO}$ & $T_{v}(\mathrm{eV})$ & $\mathrm{EBO}_{\sigma}$ & $\mathrm{EBO}_{\pi}$ & $\mathrm{EBO}$ \\
\hline \multirow[t]{3}{*}{$\mathrm{NEVPT}^{\mathrm{a}}$} & ${ }^{3} \Sigma^{-}$ & 0.00 & 0.93 & 0.87 & 1.80 & 0.00 & 0.92 & 0.85 & 1.76 \\
\hline & ${ }^{1} \Delta$ & 0.57 & 0.94 & 0.80 & 1.74 & 0.58 & 0.92 & 0.77 & 1.69 \\
\hline & ${ }^{1} \Sigma^{+}$ & 1.05 & 0.94 & 0.74 & 1.68 & 1.05 & 0.92 & 0.70 & 1.62 \\
\hline \multirow[t]{3}{*}{ c-SOCI/NEVPT2 } & $\mathrm{X}^{+}$ & 0.00 & 0.93 & 0.86 & 1.79 & 0.00 & 0.89 & 0.82 & 1.71 \\
\hline & a 1 & 0.08 & 0.93 & 0.86 & 1.79 & 0.43 & 0.89 & 0.77 & 1.66 \\
\hline & a 2 & 0.72 & 0.93 & 0.78 & 1.72 & 0.99 & 0.89 & 0.69 & 1.58 \\
\hline \multirow[t]{3}{*}{ uc-SOCI/NEVPT2 } & $\mathrm{X}^{+}$ & 0.00 & 0.90 & 0.89 & 1.78 & 0.00 & 0.86 & 0.87 & 1.73 \\
\hline & a 1 & 0.10 & 0.90 & 0.88 & 1.78 & 0.56 & 0.86 & 0.83 & 1.69 \\
\hline & a 2 & 0.74 & 0.87 & 0.87 & 1.74 & 1.09 & 0.84 & 0.79 & 1.63 \\
\hline
\end{tabular}

${ }^{a}$ CASSCF and NEVPT2 EBOs are here equivalent.

character. Note that these $\pi \rightarrow \pi^{*}$ excitations are symmetry allowed, due to the absence of a symmetry center. The $a 2 \mathrm{SO}$ state is dominated by the ${ }^{1} \Delta$ components (by more than $98 \%$ for $\mathrm{IO}^{+}$, and by about $85 \%$ for $\mathrm{AtO}^{+}$); as a result the EBOs values are marginally changed by SOC. Therefore, the contraction in SOCI calculations is in this case not expected to significantly modify the computed EBOs. We note that the SOF-EBOs and SOC-EBOs are consistent with the relative stabilities of the states: the larger the EBO, the more stable the state is. For instance, at the c-SOCI/NEVPT2 level, the SOC-EBO values of the X $0^{+}, a 1$, and $a 2$ states of $\mathrm{AtO}^{+}$are $1.71,1.66$, and 1.58 , respectively. Since the $\mathrm{X} 0^{+}$and a 1 states essentially originate from the same SOF state $\left({ }^{3} \Sigma^{-}\right)$it is not surprising to see that they exhibit close EBO values, in contrast to the $a 2$ state which originates from the higher-energy ${ }^{1} \Delta \mathrm{SOF}$ state. It is also important to stress that SOC diminishes the EBOs, notably for the ground state, which pairs with the lengthening of interatomic distance and the decrease of the harmonic frequency upon inclusion of SOC. This detailed analysis lead us to the conclusion that the EBO is a good quantitative probe of the bond nature and 
strength in molecules and of the factors that act on it.

One can now distinguish the two sets of case studies; in $I_{2}$ and $A t_{2}$, the SOC could significantly lower the EBOs since it could strongly mix the ground SOF spin components with spin components of excited SOF configurations that are less bonding, while in the $\mathrm{IO}^{+}$and $\mathrm{AtO}^{+}$cases, SOC essentially mixes the ground SOF configuration components with excited SOF components having similar EBOs. Also, the heavier the atom, the more important are SOC effects. Therefore, in iodine compounds, the SOC is not expected to drastically affect chemical bonding, contrary to what may happen in At compounds. In any case, SOC significantly affects the vertical energy excitations, and accurate determination of energy levels requires to account for all relativistic effects.

\section{CONCLUDING REMARKS}

In this work, we have shown the suitability of two-step SOCI approaches to both extend the definition of bond order analysis and obtain the energetics and spectroscopic constants for species in which SOC is important. While SOC-EBOs can safely be obtained with both c-SOCI and uc-SOCI since for the systems under consideration we have observed that both approaches yield the same qualitative picture, it should be stressed that uc-SOCI typically leads to more accurate results concerning energetics and spectroscopic data for $6 p$-containing species. The c-SOCI scheme is nevertheless appealing from the viewpoint of the EBO analysis, which allows one to easily quantify the role of SOC on chemical bonding.

For instance, for $\mathrm{At}_{2}$ the c-SOCI EBOs clearly show that SOC significantly weakens the covalent character of the chemical bond, an effect that is paired with a weakening of the bond strength. Actually, the reduction of the EBO induced by SOC is larger than the one induced by electron correlation. This emblematic case therefore demonstrates that SOC can play a more important role than electron correlation on chemical bonding. Furthermore, while it is beyond dispute that one has to go beyond the Hartree-Fock picture to determine EBOs, our results show that SOC must be considered as well to determine EBOs when heavy atoms are involved.

One should also stress that, for the diatomics under consideration, the SOC-EBO analysis was found to be relatively insensitive to the bond lengths, something that opens the perspective of using different levels of approximation when treating larger systems - for 
instance, one could perform geometry optimizations with one- or two-component DFT and perform the SOC-EBO analysis at the resulting geometries with a more sophisticated electronic structure approach.

\section{ACKNOWLEDGMENTS}

This work has been supported by grants funded by the French National Agency for Research (ANR-2010-BLAN-0807), with "Investissements d'Avenir" (ANR-11-EQPX-0004, ANR-11-LABX-0018, ANR-11-LABX-0005), and by the "Région Pays de la Loire" (NUCSAN project). This work was performed using HPC resources from CCIPL ("Centre de Calcul Intensif des Pays de la Loire"), from IDRIS-CNRS ("Institut de Développement et de Ressources en Informatique Scientifique du Centre National de la Recherche Scientifique"; contract 71859) and from CCRT-CEA ("Centre de Calcul et de Recherche Technologique", grants 2013-081859 and 2014-081859).

\section{REFERENCES}

${ }^{1}$ K. G. Dyall and K. Faegri Jr, Introduction to Relativistic Quantum Chemistry (Oxford University Press, New York, 2007).

${ }^{2}$ B. O. Roos, A. C. Borin, and L. Gagliardi, Angew. Chem. Int. Ed. 46, 1469 (2007).

${ }^{3}$ R. F. W. Bader, Chem. Rev. 91, 893 (1991).

${ }^{4}$ J. Pilmé, E. Renault, F. Bassal, M. Amaouch, G. Montavon, and N. Galland, J. Chem. Theory Comput. 10, 4830 (2014).

${ }^{5}$ A. Becke and K. E. Edgecombe, J. Chem. Phys. 92, 5397 (1990).

${ }^{6}$ B. Silvi and A. Savin, Nature 371, 683 (1994).

${ }^{7}$ M. Kohout and A. Savin, Int. J. Quant. Chem. 60, 875 (1996).

${ }^{8}$ F. Feixas, E. Matito, M. Duran, M. Solà, and B. Silvi, J. Chem. Theory Comput. 6, 2736 (2010).

${ }^{9}$ J. Pilmé, E. Renault, T. Ayed, G. Montavon, and N. Galland, J. Chem. Theory Comput. 8, 2985 (2012).

${ }^{10}$ D. S. Wilbur, Nat. Chem. 5, 246 (2013).

${ }^{11}$ D. S. Wilbur, Curr. Radiopharm. 1, 144 (2008). 
${ }^{12}$ S. Rothe, A. N. Andreyev, S. Antalic, A. Borschevsky, L. Capponi, T. E. Cocolios, H. De Witte, E. Eliav, D. V. Fedorov, V. N. Fedosseev, D. A. Fink, S. Fritzche, L. Ghys, M. Huyse, N. Imai, U. Kaldor, Y. Kudryavtsev, U. Köster, J. F. W. Lane, J. Lassen, V. Liberati, K. M. Lynch, B. A. Marsh, K. Nishio, D. Pauwels, V. Pershina, L. Popescu, T. J. Procter, D. Radulov, S. Raeder, M. M. Rajabali, E. Rapisarda, R. E. Rossel, K. Sandhu, M. D. Seliverstov, A. M. Sjödin, P. Van den Bergh, P. Van Duppen, M. Venhart, Y. Wakabayashi, and K. D. A. Wendt, Nat. Commun. 4, 1835 (2013).

${ }^{13}$ J. Champion, C. Alliot, E. Renault, B. M. Mokili, M. Chérel, N. Galland, and G. Montavon, J. Phys. Chem. A 114, 576 (2010).

${ }^{14}$ A. Sabatié-Gogova, J. Champion, S. Huclier, N. Michel, F. Pottier, N. Galland, Z. Asfari, M. Chérel, and G. Montavon, Anal. Chim. Acta 721, 182 (2012).

${ }^{15}$ J. Champion, A. Sabatié-Gogova, F. Bassal, T. Ayed, C. Alliot, N. Galland, and G. Montavon, J. Phys. Chem. A 117, 1983 (2013).

${ }^{16}$ A. Hermann, R. Hoffmann, and N. W. Ashcroft, Phys. Rev. Lett. 111, 116404 (2013).

${ }^{17}$ J. Champion, C. Alliot, S. Huclier, D. Deniaud, Z. Asfari, and G. Montavon, Inorg. Chim. Acta 362, 2654 (2009).

${ }^{18}$ J. Champion, M. Seydou, A. Sabatié-Gogova, E. Renault, G. Montavon, and N. Galland, Phys. Chem. Chem. Phys. 13, 14984 (2011).

${ }^{19}$ C. de Graaf and C. Sousa, Int. J. Quantum Chem. 106, 2470 (2006).

${ }^{20}$ R. Maurice, R. Bastardis, C. de Graaf, N. Suaud, T. Mallah, and N. Guihéry, J. Chem. Theory Comput. 5, 2977 (2009).

${ }^{21}$ J.-B. Rota, S. Knecht, T. Fleig, D. Ganyushin, T. Saue, F. Neese, and H. Bolvin, J. Chem. Phys. 135, 114106 (2011).

${ }^{22}$ L. F. Chibotaru and L. Ungur, J. Chem. Phys. 137, 064112 (2012).

${ }^{23}$ F. Gendron, D. Páez-Hernández, F.-P. Notter, B. Pritchard, H. Bolvin, and J. Autschbach, Chem. Eur. J. 20, 7994 (2014).

${ }^{24}$ F. Gendron, B. Pritchard, H. Bolvin, and J. Autschbach, Inorg. Chem. 53, 8577 (2014). ${ }^{25}$ M. Reiher and A. Wolf, Relativistic Quantum Chemistry (Wiley-VCH, Weinheim, 2009). ${ }^{26}$ B. A. Hess, C. M. Marian, and S. D. Peyerimhoff, in Advanced Series in Physical Chemistry - Vol. 2, Modern Structure Theory Part I (World Scientific, Singapore, 1995) pp. 152-278. ${ }^{27}$ F. Rakowitz and C. M. Marian, Chem. Phys. 225, 223 (1997).

${ }^{28}$ V. Vallet, L. Maron, C. Teichteil, and J.-P. Flament, J. Chem. Phys. 113, 1391 (2000). 
${ }^{29}$ F. Réal, V. Vallet, J.-P. Flament, and J. Schamps, J. Chem. Phys. 125, 174709 (2006).

${ }^{30}$ N. Douglas and N. M. Kroll, Ann. Phys. 82, 89 (1974).

${ }^{31}$ B. A. Hess, Phys. Rev. A 33, 3742 (1986).

${ }^{32}$ G. Jansen and B. A. Hess, Phys. Rev. A 39, 6016 (1989).

${ }^{33}$ D. A. Pantazis, X.-Y. Chen, C. R. Landis, and F. Neese, J. Chem. Theory Comput. 4, 908 (2008).

${ }^{34}$ D. A. Pantazis and F. Neese, Theor. Chem. Acc. 131, 1292 (2012).

${ }^{35}$ R. Ahlrichs and K. May, Phys. Chem. Chem. Phys. 2, 943 (2000).

${ }^{36}$ F. Weigend and R. Ahlrichs, Phys. Chem. Chem. Phys. 7, 3297 (2005).

${ }^{37}$ F. Neese; ORCA - An Ab Initio, Density Functional and Semiempirical Program Package (2013), version 3.0.1. Max-Planck-Institut für Bioanorganische Chemie, Mülheim an der Ruhr.

${ }^{38}$ B. O. Roos, R. Lindh, P.-Å. Malmqvist, V. Veryazov, and P.-O. Widmark, J. Phys. Chem. A 108, 2851 (2004).

39 "MOLPRO, a package of ab initio programs," (2012), version 2012.1, written by H.-J. Werner, P. J. Knowles, G. Knizia, F. R. Manby, M. Schütz, P. Celani, T. Korona, R. Lindh, A. Mitrushenkov, G. Rauhut, K. R. Shamasundar, T. B. Adler, R. D. Amos, A. Bernhardsson, A. Berning, D. L. Cooper, M. J. O. Deegan, A. J. Dobbyn, F. Eckert, E. Goll, C. Hampel, A. Hesselmann, G. Hetzer, T. Hrenar, G. Jansen, C. Köppl, Y. Liu, A. W. Lloyd, R. A. Mata, A. J. May, S. J. McNicholas, W. Meyer, M. E. Mura, A. Nicklass, D. P. O'Neill, P. Palmieri, D. Peng, K. Pflüger, R. Pitzer, M. Reiher, T. Shiozaki, H. Stoll, A. J. Stone, R. Tarroni, T. Thorsteinsson, and M. Wang (see http://www.molpro.net).

${ }^{40}$ F. Aquilante, L. De Vico, N. Ferré, G. Ghigo, P.-Å. Malmqvist, P. Neogrády, T. B. Pedersen, M. Pitonak, M. Reiher, B. O. Roos, L. Serrano-Andrés, M. Urban, V. Veryazov, and R. Lindh, J. Comput. Chem. 31, 224 (2010).

${ }^{41}$ B. O. Roos, P. R. Taylor, and P. E. M. Siegbahn, Chem. Phys. 48, 157 (1980).

${ }^{42}$ B. O. Roos, in Theory and applications of computational chemistry: The first forty years, edited by C. E. Dykstra, G. Frenking, K. S. Kim, and G. E. Scuseria (Elsevier, Amsterdam, 2005) Chap. 25, pp. 725-764.

${ }^{43}$ A. S. P. Gomes, F. Réal, N. Galland, C. Angeli, R. Cimiraglia, and V. Vallet, Phys. Chem. Chem. Phys. 16, 9238 (2014).

${ }^{44}$ B. A. Hess, C. M. Marian, U. Wahlgren, and O. Gropen, Chem. Phys. Lett. 251, 365 
(1996).

${ }^{45}$ F. Neese, J. Chem. Phys. 122, 034107 (2005).

${ }^{46}$ C. Teichteil, M. Pélissier, and F. Spiegelmann, Chem. Phys. 81, 273 (1983).

${ }^{47}$ R. Llusar, M. Casarrubios, Z. Barandiarán, and L. Seijo, J. Chem. Phys. 105, 5321 (1996).

${ }^{48}$ C. Angeli, R. Cimiraglia, S. Evangelisti, T. Leininger, and J.-P. Malrieu, J. Chem. Phys. 114, 10252 (2001).

${ }^{49}$ K. G. Dyall, J. Chem. Phys. 102, 4909 (1995).

50 "AMFI, an atomic mean-field integral program," (1996), written by B. Schimmelpfennig.

${ }^{51}$ C. Angeli, S. Borini, M. Cestari, and R. Cimiraglia, J. Chem. Phys. 121, 4043 (2004).

52 "DIRAC, a relativistic ab initio electronic structure program," (2011), release DIRAC11, revision ab65b36, written by R. Bast, H. J. Aa. Jensen, T. Saue, and L. Visscher, with contributions from V. Bakken, K. G. Dyall, S. Dubillard, U. Ekström, E. Eliav, T. Enevoldsen, T. Fleig, O. Fossgaard, A. S. P. Gomes, T. Helgaker, J. K. Lærdahl, J. Henriksson, M. Iliaš, Ch. R. Jacob, S. Knecht, C. V. Larsen, H. S. Nataraj, P. Norman, G. Olejniczak, J. Olsen, J. K. Pedersen, M. Pernpointner, K. Ruud, P. Sałek, B. Schimmelpfennig, J. Sikkema, A. J. Thorvaldsen, J. Thyssen, J. van Stralen, S. Villaume, O. Visser, T. Winther, and S. Yamamoto (see http://dirac.chem.vu.nl).

${ }^{53}$ C. E. Moore and J. Gallagher, eds., Tables of Spectra of Hydrogen, Carbon, Nitrogen, and Oxygen Atoms and Ions (CRC Press, Boca Raton, 1993).

${ }^{54}$ E. Luc-Koenig, C. Morillon, and J. Vergès, Phys. Scr. 12, 199 (1975).

${ }^{55}$ T. Fleig and A. J. Sadlej, Phys. Rev. A 65, 032506 (2002).

${ }^{56}$ I. Kim and Y. S. Lee, J. Chem. Phys. 139, 134115 (2013).

${ }^{57}$ W. C. Martin and C. H. Corliss, J. Res. Nat. Bur. Stand., Sect. A 64, 443 (1960).

${ }^{58}$ C. Morillon and J. Vergès, Phys. Scr. 12, 145 (1975).

${ }^{59}$ C. Morillon and J. Vergès, Phys. Scr. 12, 129 (1975).

${ }^{60}$ G. W. Charles, J. Opt. Soc. Am. 56, 1292 (1966).

${ }^{61}$ C. Teichteil and M. Pelissier, Chem. Phys. 180, 1 (1994).

${ }^{62}$ L. Visscher and K. G. Dyall, J. Chem. Phys. 104, 9040 (1996).

${ }^{63}$ O. Fossgaard, O. Gropen, M. Corral Valero, and T. Saue, J. Chem. Phys. 118, 10418 (2003).

${ }^{64}$ T. Nakajima and K. Hirao, J. Chem. Phys. 119, 4105 (2003).

${ }^{65}$ A. V. Mitin and C. van Wüllen, J. Chem. Phys. 124, 064305 (2006). 
${ }^{66}$ S. Höfener, R. Ahlrichs, S. Knecht, and L. Visscher, ChemPhysChem 13, 3952 (2012).

${ }^{67}$ K. P. Huber and G. Herzberg, Constants of Diatomic Molecules (Nostrand Reinhold, New York, 1979).

${ }^{68}$ See Supplementary Material Document No. for spectroscopic constants and effective bond orders computed with the SARC-DKH-TZVP basis sets.

${ }^{69}$ T. Ayed, M. Seydou, F. Réal, G. Montavon, and N. Galland, J. Phys. Chem. B 117, 5206 (2013).

${ }^{70}$ T. Ayed, F. Réal, G. Montavon, and N. Galland, J. Phys. Chem. B 117, 10589 (2013). 